\title{
Older and More Experienced? Comparing Mainland Chinese International Students in Canada on Social Cognitive Correlates of Leisure Time Physical Activity, Acculturation, and Mental Health by Study Year
}

\author{
Kimberley D. Curtin, Ph.D. \\ University of Alberta, School of Public Health \\ Tanya R. Berry, Ph.D. \\ University of Alberta, Faculty of Kinesiology, Sport, and Recreation \\ Gordon J. Walker, Ph.D.
}

University of Alberta, Faculty of Kinesiology, Sport, and Recreation

\begin{abstract}
Background: Physical activity is a mandatory component of many university programs in China and there is evidence that Chinese students value physical activity for health (Chen \& Liu, 2008); however, academics are generally prioritized as students advance in their studies (Ha, Macdonald, \& Pang, 2010). Among international students in the United States, Asian students were found to have some of the lowest rates of physical activity, which could be linked with many combinations of cultural, environmental, and individual barriers (Yoh, Yang, \& Gordon, 2008). Physical activity may aid in healthy transitions for Chinese international students.

Aim: Chinese international students were compared by study year on physical activity, mental health, culture, and social cognitive theory variables.

Method: First year $(n=92)$, second year $(n=52)$, and senior $(n=77)$ international students studying in Canada were compared on physical activity, self-efficacy, intention, access, stress, acculturative stress, subjective well-being, acculturation, and self-construal. The relationships between the social cognitive, mental health, culture variables, and physical activity were also compared.

Results: Senior students perceived fewer places to do physical activity and more discrimination compared to first- and second-year students. Senior students experienced more academic pressure and guilt towards their family compared to second year students. First year students reported more general stress compared to second year students. Self-efficacy, intention, and exercise to reduce stress shared relationships with vigorous physical activity. Subjective well-being was related to moderate physical activity, and self-efficacy and independent self-construal were related to walking. Walking decreased by study year.

Conclusions: Physical activity may be used to improve mental health for Chinese international students, but interventions may need to be adjusted or implemented depending on the academic stage of the student.
\end{abstract}

Submitted 10 July 2019: accepted 4 September 2019

Keywords: exercise, culture, international student, adjustment, leisure, mental health 
Transitioning to another country to pursue post-secondary education can present unique challenges that may change over time. Indeed, some research has shown that adaptation is most difficult in the early stages of transition for international students (Ward \& Kennedy, 1999). An initial decline in mental health, however, may improve over time following a U-shaped pattern (Zheng \& Berry, 1991). One strategy that may help ease international students' transition is physical activity, which could improve adaptation and alleviate stress (Allen, Drane, Byon, \& Mohn, 2010; Brunette, Lariviere, Schinke, Xing, \& Pickard, 2011). Physical activity is a mandatory component of many university programs in China and there is evidence that Chinese students value physical activity for health (Chen \& Liu, 2008); however, academics are generally prioritized as students advance in their studies (Ha, Macdonald, \& Pang, 2010). Among international students in the United States, Asian students were found to have some of the lowest rates of physical activity, which could be linked with many combinations of cultural, environmental, and individual barriers (Yoh, Yang, \& Gordon, 2008).

As international students spend more time in, and adapt to their host country, many will experience acculturation to mainstream culture in some capacity (Berry, 1997). Acculturation is positively associated with participation in leisure time physical activity for immigrants in general (Gerber, Barker, \& Pühse, 2012) and this pattern may hold with international students. In addition to acculturation, we can also understand behavior and cognition through selfconstrual (Markus \& Kitayama, 1991). This is how one perceives themselves in relation to others and the greater community. Self-construal influences cognitions, motivations, and emotions. Eastern cultures (e.g., Chinese, Japanese) are generally more interdependent, which means they value group harmony, and believe that people are not separate from one another. Independent cultures (e.g., United States, Australia), value the promotion of individual goals and self-assertion. The individual's thoughts and emotions give meaning to their behavior. For example, in a sport context, research on constraints to participating in skiing showed that interdependent Chinese Canadians had more interpersonal constraints, demonstrating an emphasis on social benefits of participation (Hudson, Walker, Simpson, $\&$ Hinch, 2013). Trends of acculturation and self-construal have not been analyzed in international student samples, nor the correlates and mental health outcomes related to physical activity. These variables can offer more insight into behavioral motivation and how changes in culture may be related to behavior.

In this study we used social cognitive theory (SCT; Bandura, 1986) to help explain physical activity in Chinese international students. The basis of SCT is triadic reciprocal causality, which describes behavioral outcomes as the interplay of the person, their environment, and behavior. Self-efficacy (i.e., situation specific self-confidence) has been repeatedly shown as a main correlate of physical activity behavior (Bandura, 2004). Other correlates of physical activity are outlined in SCT such as self-regulation (e.g., strategies designed to direct behavior such as goal setting), social support (e.g., friend groups), and sociostructural factors (e.g., barriers or facilitators such as access to facilities; Bandura, 2004). Factors such as acculturation and self-construal may be related to these social cognitive variables in addition to, and in conjunction with, year of study.

Physical activity can have benefits for international students, including reducing stress and improving happiness (Guo \& Ross, 2014). This may be done through cultivating social connections, improving fitness, dealing with stressors, and helping with cross-cultural transitions (Brunette et al., 2011). There is also evidence that stress can reduce physical activity, meaning that mental health and physical activity are likely interrelated (Stults-Kolehmainen $\&$ Sinha, 2014). Many international students may feel they lack time to be physically active due to competing leisure activities or academic work, lack information about activities, or inconvenient facilities (Guo \& Ross, 2014; Hashim, 2012). 
The purpose of this study was to examine the differences that may be present by study year in leisure time physical activity, and the social cognitive, cultural, and mental health correlates of physical activity among Chinese international students studying in Canada. To date, differences in physical activity and these important behavioral correlates have not been examined at different stages of study for mainland Chinese international students, and more effective physical activity promotion could be implemented if it is tailored for stage of study.

\section{METHODS}

\section{Recruitment}

After obtaining ethical approval from the University Research Ethics Board mainland, Chinese international students were recruited via social media posts, posters, in class announcements, email newsletters for international students, in-person on campus, or through a psychology research pool for course credit. All the participants were Chinese citizens studying in Canada on a study permit. Students were directed to the online survey via the SoJump.com survey platform where they read an information letter and proceeded with the study at their discretion. The university where the students study is very similar to a 4-year College in the United States of America where students engage in campus life in addition to academics during their study period.

\section{Measures}

All measures were translated into simplified Chinese by three bilingual graduate students from China using a committee approach (Marín \& Marín, 1991) and administered to a large sample of Chinese students at a large university in South Eastern China. Exploratory factor analyses (EFA) were then conducted, and the measures were adjusted based on correlations, factor loadings, and consultations with the translators. Any changes made to the original questionnaires were reflected in the following paragraphs.

Social Cognitive Theory Variables. Self-Efficacy was assessed using the nine-item Multidimensional Exercise Self-Efficacy Scale (MSES; Rodgers, Wilson, Hall, Fraser, \& Murray, 2008) which measures task (i.e., performing physical movements), coping (i.e., overcoming challenges), and scheduling (i.e., planning effectively) self-efficacy. Rodgers and colleagues (2008) found that these items were reliable in undergraduate students (task $\alpha=.81$, coping $\alpha$ $=.81$, scheduling $\alpha=.91)$. The EFA supported a single factor $(\alpha=.94)$. Intention was measured using one question assessing how likely one was to regulate their physical activity behaviour (Karoly, 1993; Luszczynska \& Schwarzer, 2005). Social support was measured using The Friend Support for Exercise Habits Scale (Sallis, Grossman, Pinski, Patterson, \& Nader, 1987) which includes five items that measure frequency of social support for exercise during the past week. Good internal consistency has been reported; $\alpha=.91$ (Rovniak, Anderson, Winett, \& Stephens, 2002). Two items were used to assess the sociostructural barrier or facilitator of perceived access to facilities to be physically active inside and outside the University.

Culture Variables. The Vancouver Index of Acculturation (VIA; Ryder, Alden, \& Paulhus, 2000) was used to examine acculturation. The VIA includes 20 items which measure how one identifies and participates in their heritage cultural practices (i.e., Chinese, 10 items) as well as typical mainstream (i.e., Canadian, 10 items) practices independently. The heritage and mainstream subscales have shown internal consistencies in Chinese undergraduate samples of $\alpha=.91$, and $\alpha=.89$, respectively (Huynh, Howell, \& Benet-Martinez, 2009). Self-construal was measured 
using a rating of agreement on 16-items (Park \& Kitayama, 2012). The original instruments had 20 items, but one item from the independent subscale, and three items from the interdependent subscale were removed following the EFA. The scale yielded scores for interdependent and independent self-construal and has shown internal consistencies of $\alpha=.66$ and $\alpha=.78$, respectively for a sample of Japanese, Asian Canadians, and European Canadians (Ito, 2014).

Mental Health Variables. The Acculturative Stress Scale for Chinese College Students in the United States (Altered to reference Canada; Bai, 2016) is a 32-item, five-factor measure of frequency of language insufficiency (10 items), social isolation ( 8 items), perceived discrimination (7 items), academic pressure (4 items), and guilt towards family (3 items). The measure had internal consistency of $\alpha=.93$ in a sample of Chinese students in the United States (Bai, 2016). The Perceived Stress Scale - 10 item (Cohen, Kamarck, \& Mermelstein, 1983) measured frequency of certain thoughts or feelings in the past week to positive and negative perceptions of life events. The two-factor structure was supported in an adult sample of Chinese women, and had an overall reliability of $\alpha=.86$ (Wang et al., 2011).

Subjective well-being (SWB) was assessed according to Diener \& Ryan (2009) who suggested that SWB includes frequent positive emotions, infrequent negative emotions, and high life satisfaction. The life satisfaction component was assessed using the Satisfaction with Life Scale (Diener, Emmons, Larsen, \& Griffin, 1985). There were originally five items rated on a 7-point scale from 1 (strongly disagree) to 7 (strongly agree) that assess overall life satisfaction. In a sample of Hong Kong university students, the single-factor model was supported, with a reliability of $\alpha=.82$ (Sachs, 2003). The final item was dropped due to low factor loadings in the EFA. Items from the Affect Valuation Index (Tsai, Knutson, \& Fung, 2006) were used to assess the affective portion of SWB (actual affect only). Participants rated frequency of positive ( 8 items; $\alpha=.87$ ), negative ( 9 items; $\alpha=.83$ ), and low arousal positive ( 3 items; $\alpha=.79$ ) emotions from 1 (never) to 5 (all the time). Satisfaction with life scores were added to the positive emotion frequencies, and negative emotions were subtracted from this total to generate a composite SWB score.

The stress management subscale (four items) from the Exercise Motivations Inventory II (Markland \& Ingledew, 1997) was used to assess the use of exercise as a coping mechanism for stress. The subscale had an internal reliability of $\alpha$ $=.92$ (Markland \& Ingledew, 1997), and was found to be acceptable in a diverse sample of college students in the United States (Egli, Bland, Melton, \& Czech, 2011). During translation the final item was dropped from the survey due to redundancy.

Leisure Time Physical Activity. The Chinese version of the International Physical Activity Questionnaire- short version (IPAQ-C) was used to assess self-reported physical activity over the last seven days. Days per week and hours/minutes per day of vigorous physical activity (VPA; e.g., heavy lifting, aerobics), moderate physical activity (MPA; e.g., doubles tennis, regular biking), walking, and sitting were reported. Days and minutes of each activity type were multiplied by their respective quotient $(\mathrm{VPA}=8.0, \mathrm{MPA}=4.0$, walking $=3.3)$ to calculate metabolic equivalents of a task (METs). The Chinese version has been found to be reliable (interclass correlation coefficient $=.079$ ) and showed agreement with a week-long physical activity log (Macfarlane, Lee, Ho, Chan, \& Chan, 2007).

Demographics. Questions measuring age, sex, height, weight, program of study, and year of study were included. Height and weight were used to calculate body mass index (BMI) in kilograms per meter squared.

\section{Participants}

There were 221 students included in the sample (56.1\% female; Table 1). There were 92 first year students, 52 second year students, and 77 senior students ( $3^{\text {rd }}$ year or higher). The senior students were combined because they were not 
different on the outcome measures of interest or descriptive variables. Most senior students were in their third year of study $(n=49)$, or their fourth year of study $(n=26)$, while a few were doing a fifth year $(n=2)$. Students were studying economics $(24.7 \%)$, psychology $(18.5 \%)$, computer science $(12.9 \%)$, science $(11.2 \%)$, engineering $(11.2 \%)$ and several other subjects such as agriculture, arts, math, business, and languages. A power analysis using the $G^{*}$ power computer program (Faul, Erdfelder, Lang, \& Buchner, 2007) indicated that a total maximum sample of 120 people would be needed to detect medium effects $\left(\mathrm{f}^{2}=.15\right)$ with $95 \%$ power using a MANOVA to examine differences in means between student groups on physical activity, social cognitive, mental health, and culture variables with alpha at .05. For the regression models, a total sample size of 217 was recommended to detect a medium effect of social cognitive, mental health, and culture variables on VPA, MPA, and walking $\left(\mathrm{f}^{2}=.15\right)$ with $95 \%$ power using an alpha of .05.

Table 1

Descriptive Statistics by Study Year

\begin{tabular}{|c|c|c|c|c|c|c|}
\hline Variable & First Year & & Second Year & & Senior & \\
\hline & Mean (SD) & Range & Mean (SD) & Range & Mean (SD) & Range \\
\hline VPA & $830.96(1255.83)$ & $0-4944$ & $\begin{array}{l}910.46 \\
(1276.85)\end{array}$ & 0.4944 & $\begin{array}{l}630.75 \\
(1030.86)\end{array}$ & $0-3840$ \\
\hline MPA & $522.54(667.43)$ & $0-2906$ & $\begin{array}{l}504.43 \\
(761.81)\end{array}$ & $0-2906$ & $\begin{array}{l}433.62 \\
(649.61)\end{array}$ & $0-2906$ \\
\hline Walking & $1374.68(913.16)$ & $0.00-4158.0$ & $\begin{array}{l}1177.86 \\
(1020.84)\end{array}$ & $0-4776$ & $\begin{array}{l}1014.82 \\
(948.10)\end{array}$ & $0-4158$ \\
\hline Self-efficacy & $53.11(24.43)$ & $0-96.67$ & $54.51(20.06)$ & $8.67-95.00$ & $49.05(24.33)$ & $0-100$ \\
\hline Intention & $4.55(1.91)$ & $1.00-7.00$ & $4.44(1.93)$ & $1.00-7.00$ & $4.18(1.97)$ & $1.00-7.00$ \\
\hline Social support & $1.83(1.83)$ & $1.00-4.80$ & $1.91(0.90)$ & $1.00-4.60$ & $1.96(0.96)$ & $1.00-5.00$ \\
\hline $\begin{array}{l}\text { Perceived } \\
\text { access }\end{array}$ & $4.27(1.31)^{\mathrm{b}}$ & $1.00-7.00$ & $4.57(1.43)^{\mathrm{b}}$ & $1.50-7.00$ & $3.78(1.31)^{\mathrm{a}}$ & $1.00-7.00$ \\
\hline $\begin{array}{l}\text { Chinese } \\
\text { acculturation }\end{array}$ & $6.77(1.39)$ & $1.60-9.00$ & $6.91(1.41$ & $3.00-8.90$ & $6.89(1.11)$ & $3.40-8.80$ \\
\hline $\begin{array}{l}\text { Mainstream } \\
\text { acculturation }\end{array}$ & $5.84(1.07)$ & $1.60-8.40$ & $5.93(1.12$ & $3.20-8.00$ & $5.80(1.19)$ & $1.70-8.40$ \\
\hline Independent SC & $3.51(0.53)$ & $1.67-4.89$ & $3.53(0.61)$ & $2.00-5.00$ & $3.56(0.66)$ & $1.78-5.00$ \\
\hline $\begin{array}{l}\text { Interdependent } \\
\text { SC }\end{array}$ & $4.10(0.56)$ & $2.29-5.00$ & $3.88(0.65)$ & $2.00-5.00$ & $4.04(0.59)$ & $2.71-5.00$ \\
\hline $\begin{array}{l}\text { Language } \\
\text { insufficiency }\end{array}$ & $3.49(1.42)$ & $1.00-7.00$ & $3.17(1.18)$ & $1.20-5.60$ & $3.17(1.17)$ & $0.91-6.27$ \\
\hline Social isolation & $3.06(1.21)$ & $1.22-7.00$ & $3.29(1.27)$ & $1.00-6.50$ & $3.46(1.13)$ & $1.00-5.88$ \\
\hline $\begin{array}{l}\text { Guilt Towards } \\
\text { family }\end{array}$ & $3.291 .61)^{\mathrm{ab}}$ & $1.00-7.00$ & $3.10(1.66)^{b}$ & $1.00-7.00$ & $3.81(1.63)^{\mathrm{a}}$ & $1.00-7.00$ \\
\hline Discrimination & $2.19(1.04)^{b}$ & $1.00-5.83$ & $2.43(1.39)^{\mathrm{b}}$ & $1.00-6.83$ & $2.98(1.17)^{a}$ & $1.00-5.29$ \\
\hline
\end{tabular}




\begin{tabular}{lllllll}
\cline { 1 - 5 } $\begin{array}{l}\text { Academic } \\
\text { pressure }\end{array}$ & $3.61(1.55)^{\mathrm{ab}}$ & $1.00-7.00$ & $3.33(1.52)^{\mathrm{b}}$ & $1.00-7.00$ & $3.99(1.57)^{\mathrm{a}}$ & $1.50-7.00$ \\
General stress & $30.10(5.93)^{\mathrm{a}}$ & $17.00-47.00$ & $27.56(5.58)^{\mathrm{b}}$ & $15.00-41.00$ & $29.09(5.32)^{\mathrm{ab}}$ & $17.00-42.00$ \\
SWB & $7.92(2.88)$ & $-0.79-13.28$ & $7.86(2.74)$ & $0.71-12.64$ & $7.50(2.30)$ & $2.43-12.72$ \\
Exercise to & $4.32(1.38)$ & $1.00-6.00$ & $4.10(1.34)$ & $1.00-6.00$ & $4.14(1.32)$ & $1.00-6.00$ \\
cope with stress & & & & & & \\
Age & $18.92(1.20)^{\mathrm{a}}$ & $17-23$ & $19.92(1.06)^{\mathrm{b}}$ & $18-23$ & $21.53(1.62)^{\mathrm{c}}$ & $17-26$ \\
BMI & $21.49(3.33)$ & $15.60-29.30$ & $21.13(3.22)$ & $15.76-29.41$ & $21.78(4.10)$ & $15.89-40.12$ \\
\hline
\end{tabular}

Note. VPA = vigorous intensity leisure time physical activity; MPA = moderate intensity leisure time physical activity; $\mathrm{SC}=$ self-construal; $\mathrm{BMI}=$ body mass index; $\mathrm{SWB}=$ subjective well-being; $\mathrm{SD}=$ standard deviation.

Physical activity was measured in metabolic equivalents (METs), self-efficacy was rated from 0-100\%, SWB was calculated by adding positive affect and life satisfaction and then subtracting negative affect, age was reported in years, BMI was calculated using self-reported height and weight. All other measures were reported using Likert scales.

Different superscript letters indicate a significant difference between the groups $p<.05$.

\section{Data Preparation and Analysis}

Differences between the final three student groups were examined using four multivariate analyses of variance (MANOVA) models grouped together based on theoretical similarity (Table 2). The first model examined differences in VPA, MPA and walking between the groups. In the second model the SCT variables (i.e., self-efficacy, intention, access, and social support) were entered. The third model included both types of self-construal (i.e., independent and interdependent), and acculturation (i.e., mainstream and heritage), and BMI was included as a covariate in this model. The fourth model included all the mental health variables (i.e., acculturative stress subscales, general stress, SWB, and exercise as a coping strategy for stress). Follow-up tests were corrected using the Bonferroni correction. Table 3 includes bivariate correlations. If the equality of covariances assumption was violated (using Box's m-test) Hotelling's trace statistic was used (Field, 2013).

Table 2

Variables Included in Each MANOV A Model

\begin{tabular}{ll}
\hline Model & Dependent variables \\
\hline 1 & Vigorous physical activity, moderate physical activity, and walking \\
2 & Self-efficacy, intention to be physically active, access perceptions, social support \\
3 & Independent self-construal and interdependent self-construal, mainstream \\
& acculturation, heritage acculturation, body mass index (covariate) \\
4 & Language insufficiency, social isolation, discrimination, academic pressure, guilt \\
& towards family, general stress, subjective well-being, exercise to cope with stress \\
\hline
\end{tabular}

Note: Independent variable was student group (first year, second year, or senior) 
Three hierarchical linear regressions were used to measure relationships between culture variables (i.e., acculturation, self-construal), SCT variables, and mental health variables with physical activity as the dependent variable (i.e., VPA, MPA, walking). Variables were entered according to Field (2013) who suggests that known predictors should enter the model first followed by variables that have not previously been tested. Step 1 included year of study because this variable may be an important indicator of physical activity but may become less important compared to the other variables. Step 2 included SCT variables because these are known contributors to physical activity. Step 3 included the mental health variables which are also known to be related to physical activity. Step 4 included the culture variables to examine their contribution to physical activity beyond the SCT and mental health variables. Additionally, the moderating effect of year of study was examined in post-hoc models if it was significantly related to the dependent variable. All interaction variables were centered prior to entering them into the model.

Because the dependent variable (physical activity) is measured in METs, the unstandardized beta values were used to determine a practical effect of the independent variables by calculating minutes of physical activity gained or lost in relation to the independent variable on one day by dividing the unstandardized beta value by the MET quotient for the specific physical activity intensity. For example, using self-efficacy as the independent variable,13.05 METs (unstandardized beta value) for VPA would be divided by 8 (MET quotient) to indicate 1.63 minutes of VPA could be gained due to an increase in one unit of self-efficacy (i.e., an increase in 1\% on a $0 \%$ - 100\% scale). 
Table 3

Bivariate Correlations for all Participants

\begin{tabular}{|c|c|c|c|c|c|c|c|c|c|c|c|c|c|c|c|c|c|c|c|c|c|c|c|}
\hline & 1 & 2 & 3 & 4 & 5 & 6 & 7 & 8 & 9 & 10 & 11 & 12 & 13 & 14 & 15 & 16 & 17 & 18 & 19 & 20 & 21 & 22 & 23 \\
\hline 1.VPA & 1 & & & & & & & & & & & & & & & & & & & & & & \\
\hline 2.MPA & $.26^{\mathrm{a}}$ & 1 & & & & & & & & & & & & & & & & & & & & & \\
\hline 3.Walking & .05 & $.25^{\mathrm{a}}$ & 1 & & & & & & & & & & & & & & & & & & & & \\
\hline 4.Self-efficacy & $.46^{\mathrm{a}}$ & $.24^{\mathrm{a}}$ & $.26^{\mathrm{a}}$ & 1 & & & & & & & & & & & & & & & & & & & \\
\hline 5.Intention & $.45^{a}$ & $.18^{a}$ & $.20^{\mathrm{a}}$ & $.70^{\mathrm{a}}$ & 1 & & & & & & & & & & & & & & & & & & \\
\hline 6.Social support & $.20^{\mathrm{a}}$ & $.18^{a}$ & .04 & $.17^{\mathrm{b}}$ & $.25^{\mathrm{a}}$ & 1 & & & & & & & & & & & & & & & & & \\
\hline $\begin{array}{l}\text { 8.Chinese } \\
\text { acculturation }\end{array}$ & -.01 & .05 & .02 & .06 & .13 & .09 & .13 & 1 & & & & & & & & & & & & & & & \\
\hline $\begin{array}{l}\text { 9.Mainstream } \\
\text { acculturation }\end{array}$ & $.17^{\mathrm{b}}$ & $.17^{\mathrm{b}}$ & $.14^{\mathrm{b}}$ & $.29^{\mathrm{a}}$ & $.26^{\mathrm{a}}$ & $.22^{\mathrm{a}}$ & .10 & $.20^{\mathrm{a}}$ & 1 & & & & & & & & & & & & & & \\
\hline 10.Independent SC & .11 & $.15^{\mathrm{b}}$ & $.18^{a}$ & $.16^{\mathrm{b}}$ & -.02 & .12 & .04 & .07 & $.18^{\mathrm{a}}$ & 1 & & & & & & & & & & & & & \\
\hline $\begin{array}{l}\text { 11.Interdependent } \\
\text { SC }\end{array}$ & $.14^{\mathrm{b}}$ & $.14^{\mathrm{b}}$ & $.15^{\mathrm{b}}$ & .13 & $.21^{\mathrm{a}}$ & .08 & .13 & $.42^{\mathrm{a}}$ & $.18^{a}$ & -.06 & 1 & & & & & & & & & & & & \\
\hline $\begin{array}{l}\text { 12.Language } \\
\text { insufficiency }\end{array}$ & -.11 & -.03 & .04 & $-.16^{\mathrm{b}}$ & -.09 & $-.15^{b}$ & -.11 & $.19^{a}$ & $-.30^{a}$ & $-.22^{\mathrm{a}}$ & $.20^{\mathrm{a}}$ & & & & & & & & & & & & \\
\hline 13.Social isolation & -.10 & -.12 & -.07 & $-.14^{\mathrm{b}}$ & $-.22^{\mathrm{a}}$ & -.11 & $-.23^{a}$ & .06 & $-.27 \mathrm{a}$ & -.12 & .02 & $.58^{\mathrm{a}}$ & 1 & & & & & & & & & & \\
\hline 14.Discrimination & -.10 & .07 & .09 & -.07 & -.07 & -.02 & $-.32^{\mathrm{a}}$ & .02 & -.06 & .05 & .00 & $.41^{\mathrm{a}}$ & $.50^{\mathrm{a}}$ & 1 & & & & & & & & & \\
\hline $\begin{array}{l}\text { 15.Academic } \\
\text { pressure }\end{array}$ & -.10 & -.08 & .00 & $-.22^{\mathrm{a}}$ & $-.24^{\mathrm{a}}$ & -.05 & $-.30^{\mathrm{a}}$ & -.02 & $-.14^{\mathrm{b}}$ & -.03 & .03 & $.37^{\mathrm{a}}$ & $.51^{\mathrm{a}}$ & $.42^{\mathrm{a}}$ & 1 & & & & & & & & \\
\hline $\begin{array}{l}\text { 16.Guilt towards } \\
\text { family }\end{array}$ & .00 & .05 & .09 & -.09 & .00 & $.14^{\mathrm{b}}$ & -.08 & $.27^{\mathrm{a}}$ & .03 & -.01 & $.25^{\mathrm{a}}$ & $.30^{\mathrm{a}}$ & $.31^{\mathrm{a}}$ & $.30^{\mathrm{a}}$ & $.37^{\mathrm{a}}$ & 1 & & & & & & & \\
\hline 17.General stress & -.03 & -.02 & .09 & -.04 & -.04 & -.04 & $-.19^{a}$ & -.13 & $-.13^{\mathrm{b}}$ & -.06 & .08 & $.38^{a}$ & $.39 \mathrm{a}$ & $.34^{\mathrm{a}}$ & $.56^{\mathrm{a}}$ & $.22^{\mathrm{a}}$ & 1 & & & & & & \\
\hline 18.SWB & .11 & $.21^{\mathrm{a}}$ & .08 & .11 & .08 & .09 & $.18^{\mathrm{b}}$ & $.22^{\mathrm{a}}$ & $.15^{\mathrm{b}}$ & $.27^{\mathrm{a}}$ & .08 & $-.15^{\mathrm{b}}$ & $-.35^{\mathrm{a}}$ & $-.19^{a}$ & $-.33^{\mathrm{a}}$ & -.04 & $-.49^{a}$ & 1 & & & & & \\
\hline $\begin{array}{l}\text { 19. Exercise to } \\
\text { reduce stress }\end{array}$ & $.36^{\mathrm{a}}$ & $.23^{\mathrm{a}}$ & .09 & $.29^{a}$ & $.43^{a}$ & $.25^{\mathrm{a}}$ & $.34^{\mathrm{a}}$ & $.15^{\mathrm{b}}$ & $.35^{\mathrm{a}}$ & .06 & $.23^{\mathrm{a}}$ & .03 & -.05 & .05 & -.01 & $.19^{a}$ & .03 & .12 & 1 & & & & \\
\hline 20.Study year & -.07 & -.08 & $-.18^{\mathrm{b}}$ & -.04 & -.07 & .04 & $-.17^{b}$ & .03 & .00 & -.01 & -.08 & -.13 & $.14^{\mathrm{b}}$ & $.28^{\mathrm{a}}$ & .11 & .11 & -.06 & -.06 & -.06 & 1 & & & \\
\hline 21.Age & -.11 & -.04 & $-.18^{a}$ & .03 & -.03 & .01 & -.12 & .05 & -.01 & -.01 & -.03 & -.13 & $.15^{\mathrm{b}}$ & $.16^{\mathrm{b}}$ & .11 & .05 & -.05 & -.03 & .00 & $.71^{\mathrm{a}}$ & 1 & & \\
\hline
\end{tabular}

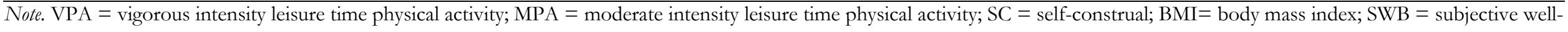
being. 


\section{RESULTS}

\section{Preliminary Analyses}

Data were first analyzed for missing data, normality, outliers, and differences between age, sex, and BMI on the independent and dependent variables. For participants who reported days of physical activity but not minutes, or vice versa, the mean minutes or days for that activity type was inserted to calculate individual METs. Outlying scores ( $z \geq$ 3.20) were recalculated to be three standard deviations above or below the mean. No multivariate outliers were identified when examined using Mahalanobis distances. Data were found to be missing at maximum $11.8 \%$ for any variable and missing completely at random according to Little's MCAR test $(p>.05)$. Skewness and kurtosis values along with visual inspection of the variable distributions indicated that MPA and walking were slightly skewed but did not warrant transformation. Participants with a higher BMI reported more independent self-construals, and age differed by study year $(\phi<.05)$. Participants were not different on age, or sex for any of the independent or dependent variables, and the effect of sex was null in the regression models. As a result, sex was not included in the models.

\section{Student Comparisons by Year}

There was no effect of study year on the physical activity or culture variables.

SCT. Using Pillai's trace, there was a significant effect of study year on the SCT variables, $V=.07, F(8,432.00)$ $=1.96, p=.05$. In particular, students were different by study year on perceptions of access to places to do physical activity, $F(2,218)=6.16, p=.002$. Follow up univariate tests showed that senior students had significantly lower perceptions of access to places to do physical activity compared to first year $(p=.05,95 \%$ CI $[-0.968--0.001], d=$ $.38)$ and second year students $(p=.003,95 \%$ CI $[-1.350--0.226], d=.62)$.

Mental Health. Using Hotelling's trace, there was a moderate effect of study year on the mental health variables, $T=.31, F(16,420.00)=4.04, p<.001$. The groups differed on perceptions of discrimination $(F(2,218)=9.77, p<$ $.001)$, academic pressure $(F(2,218)=3.04, p=.05)$, feelings of guilt towards their family $(F(2,218)=3.58, p=.03)$, and general stress $(F(2,218)=3.55, p=.03)$. Follow-up univariate tests showed that senior students rated themselves higher on discrimination compared to the first year $(p<.001,95 \% \mathrm{CI}[0.356-1.230], d=.72)$ and second year students $(p=.03,95 \%$ CI $[-0.040-1.056], d=.43)$. Second year students rated themselves lower on academic pressure compared to senior students $(p=.05,95 \%$ CI $-1.336-0.003], d=.43)$. Senior students rated themselves higher on guilt towards their family compared to second year students $(p=.04,95 \%$ CI $[0.0122-1.410], d=.44)$. Finally, first year students reported more general stress compared to second year students $(p=.03,95 \%$ CI $[0.237-4.849], d=$ .45).

\section{Relationships to Physical Activity}

Self-efficacy, intention, and exercise to reduce stress shared relationships with VPA (Table 4). For every one unit increase in self-efficacy (on a 100-unit scale), participants did 1.6 more minutes of VPA. About 15 minutes of VPA was added for a 1-point increase in intention, and 19.17 minutes were added for a 1-point increase in perceptions that exercise can reduce stress. SWB was the most important variable related to MPA (Table 5), adding about 11 minutes of MPA per unit increase in SWB. Year of study, self-efficacy, and independent self-construal were related to walking.

Walking (Table 6) decreased by about 45 minutes per study year increase. A univariate test showed that first year 
students walked significantly more than senior students $(\phi=.043)$. Every unit increase in self-efficacy was related to about 2 minutes of walking, and an increase in independent self-construal added over an hour of walking.

Table 4

Regression Model Summaries for Vigorous Physical Activity

\begin{tabular}{|c|c|c|c|}
\hline Variable & $\mathrm{B}$ & SE B & $\beta$ \\
\hline (Constant) & -1552.79 & 903.13 & \\
\hline Year of Study & -10.77 & 69.77 & -0.01 \\
\hline Self-efficacy & 13.05 & 4.06 & $0.25^{* *}$ \\
\hline Intention & 121.97 & 50.37 & $0.21 *$ \\
\hline Social Support & 75.37 & 80.41 & 0.06 \\
\hline Access & 41.02 & 58.86 & 0.05 \\
\hline Exercise to reduce stress & 153.33 & 62.08 & $0.18^{*}$ \\
\hline Language difficulty & -50.32 & 77.56 & -0.06 \\
\hline Social isolation & 63.38 & 82.44 & 0.07 \\
\hline Discrimination & -79.58 & 74.01 & -0.08 \\
\hline Academic pressure & 34.21 & 59.93 & 0.05 \\
\hline Guilt towards family & 8.69 & 48.70 & 0.01 \\
\hline General stress & -3.32 & 16.61 & -0.02 \\
\hline Subjective well-being & 20.35 & 32.08 & 0.05 \\
\hline Chinese Acculturation & -108.10 & 63.78 & -0.12 \\
\hline Canadian Acculturation & -47.76 & 73.41 & -0.04 \\
\hline Independent SC & 127.59 & 125.66 & 0.07 \\
\hline Interdependent SC & 169.04 & 133.39 & 0.09 \\
\hline
\end{tabular}

Note. Only the final step is shown. Step $1 \mathrm{R}^{2}=.004$, Step $2 \Delta \mathrm{R}^{2}=.26^{* *}$, Step $3 \Delta \mathrm{R}^{2}=.03$, Step $4 \Delta \mathrm{R}^{2}=.02$. ${ }^{*} p<.05,{ }^{* *} p<.01$. 
Table 5

Regression Model Summaries for Moderate Physical Activity

\begin{tabular}{lrlr}
\hline Variable & B & SE B & \\
\hline Constant) & -861.77 & 558.36 & -0.08 \\
Year of Study & -49.16 & 43.14 & 0.15 \\
Self-efficacy & 4.51 & 2.51 & -0.05 \\
Intention & -14.87 & 31.14 & 0.11 \\
Social Support & 77.35 & 49.71 & -0.04 \\
Access & -19.23 & 36.39 & 0.13 \\
Exercise to reduce stress & 64.04 & 38.38 & -0.01 \\
Language difficulty & -4.92 & 47.95 & -0.08 \\
Social isolation & -42.81 & 50.97 & 0.14 \\
Discrimination & 76.18 & 45.75 & -0.06 \\
Academic pressure & -26.26 & 37.05 & 0.02 \\
Guilt towards family & 6.22 & 30.11 & 0.07 \\
General stress & 8.34 & 10.27 & $0.18^{*}$ \\
Subjective well-being & 43.06 & 19.83 & -0.05 \\
Chinese Acculturation & -24.11 & 39.43 & 0.01 \\
Canadian Acculturation & 3.12 & 45.39 & 0.04 \\
Independent SC & 42.79 & 77.69 & 0.09 \\
Interdependent SC & 97.14 & 82.47 & $S t e p 4 \Delta \mathrm{R}^{2}=.01$.
\end{tabular}

Note. Only the final step is shown. Step $1 \mathrm{R}^{2}=.01$, Step $2 \Delta \mathrm{R}^{2}=.07^{* *}$, Step $3 \Delta \mathrm{R}^{2}=.07^{*}$, Step $4 \Delta \mathrm{R}^{2}=.01$. ${ }^{*} p<.05,{ }^{* *} p<.01$. 
Table 6

Regression Model Summaries for Walking

\begin{tabular}{|c|c|c|c|}
\hline Variable & $\mathrm{B}$ & SE B & $\beta$ \\
\hline (Constant) & -650.57 & 793.26 & \\
\hline Year of Study & -151.35 & 61.29 & $-0.18^{*}$ \\
\hline Self-efficacy & 7.25 & 3.57 & $0.17^{*}$ \\
\hline Intention & 43.82 & 44.24 & 0.09 \\
\hline Social Support & -33.86 & 70.62 & -0.03 \\
\hline Access & -51.45 & 51.70 & -0.07 \\
\hline Exercise to reduce stress & -29.91 & 54.53 & -0.04 \\
\hline Language difficulty & 47.76 & 68.12 & 0.07 \\
\hline Social isolation & -86.88 & 72.41 & -0.11 \\
\hline Discrimination & 76.89 & 65.00 & 0.10 \\
\hline Academic pressure & 7.11 & 52.64 & 0.01 \\
\hline Guilt towards family & 58.08 & 42.78 & 0.10 \\
\hline General stress & 7.30 & 14.59 & 0.04 \\
\hline Subjective well-being & 16.92 & 28.18 & 0.05 \\
\hline Chinese Acculturation & -62.72 & 56.02 & -0.09 \\
\hline Canadian Acculturation & 47.88 & 64.48 & 0.06 \\
\hline Independent SC & 221.86 & 110.37 & $0.14^{*}$ \\
\hline Interdependent SC & 177.29 & 117.16 & 0.11 \\
\hline
\end{tabular}

Note. Only the final step is shown. Step $1 \mathrm{R}^{2}=.03^{* *}$, Step $2 \Delta \mathrm{R}^{2}=.07^{* *}$, Step $3 \Delta \mathrm{R}^{2}=.04$, Step $4 \Delta \mathrm{R}^{2}=.03$. ${ }^{*} p<.05,{ }^{* *} p<.01$

\section{DISCUSSION}

This research aimed to describe how social cognitive, cultural, and mental health correlates of physical activity differed over time for Chinese international students in Canada by comparing a sample of first year, second year, and senior undergraduate students. Senior students appeared to have a unique experience from the second year and first year students. Senior students perceived fewer places to do physical activity, and experienced more discrimination compared to the first- and second-year students. Senior students reported more guilt towards their family and academic pressure compared to the second-year students. The initial transition to Canada appears to induce more general stress as first year students reported more stress compared to second year students. This latter finding supports work by Ward and Kennedy (1999) who indicated that initial transitions were the most difficult for international students. These trends may help identify times during a Chinese international student's degree that are critical for intervention.

No differences were found for the culture variables between the study years as anticipated. Additionally, year of study was negatively related to walking which contradicts research suggesting that more acculturation was related to more physical activity in immigrants and in qualitative research with international students (Guo \& Ross, 2014; Yan $\&$ FitzPatrick, 2016). This may simply be a result of the narrowing of course topics taken in senior years reducing the need to travel around campus. Interestingly, more similarity to mainstream culture (i.e., independent self-construal) 
was related to more walking. This self-construal is likely developed over time in combination with other factors. In a study of East Asians in Germany, those who were assimilated or integrated into the host culture were characterized by high independent self-construal, and those who were assimilated had fewer depressive symptoms relative to the other groups (Shim, Freund, Stopsack, Kämmerer, \& Barnow, 2014). As such, independent self-construal may act as an indicator of similarity to Canadian culture, but this should be tested with a representative sample. More independent students may feel more comfortable in the community and the larger cultural climate and because of this they may be more likely to participate in activities on campus or with friends.

Of the SCT variables self-efficacy was expected to share the largest relationship with physical activity based on previous research (Ayotte, Margrett, \& Hicks-Patrick, 2010). For VPA and walking, self-efficacy may be important targets for all the student groups. In addition, more VPA was related to improved intention to be physically active and a belief in physical activity to cope with stress. Intention could be considered a self-regulatory mechanism; it implies planning and behaviour management (Karoly, 1993; Luszczynska \& Schwarzer, 2005). In a sample of first year college students in the United States, self-efficacy and challenging physical activity goals were related to more VPA (Doerksen, Umstattd, \& McAuley, 2009). Improving self-efficacy may be enough to promote walking among Chinese international students, and it may be particularly important in later years of study to also improve perceptions of access. Access perceptions are hypothesized to improve intentions to do physical activity (Bandura, 2004). As such, perceptions of decreased access could hinder participation especially in the senior students.

In addition to self-efficacy, we expected that social support would be related to physical activity in the present sample. Previous qualitative work suggested that physical activity was a valuable context in that it promoted socialization with peers for international students (Li \& Zizzi, 2018). Social support was not different by study year indicating that novelty, small social circles, or adjustment are likely not the only reasons for the null effect of social support on physical activity. It is possible that social support was indirectly related to physical activity as this relationship has been previously identified (Rovniak et al., 2002). Social support can facilitate self-regulation, and increase self-efficacy (Bandura, 1997), and as such it may have played a role in the relationship between intention, self-efficacy and VPA. Future studies should use modelling techniques to identify these indirect relationships.

Asian international students may not recognize the benefits of physical activity for supporting social interaction, more often recognizing physical benefits of physical activity (Guo \& Ross, 2014). Social isolation has been linked to language insufficiency which may make students feel uncomfortable interacting with others in a physical activity setting (McLachlan \& Justice, 2009; Yeh \& Inose, 2003). Language insufficiency was correlated with almost all the other acculturative stress subscales in this study, showing that it can contribute to various types of stress for undergraduate students. Social relationships and facilitators of these relationships (i.e., language training, integration events) should be fostered in university environments. Furthermore, other acculturative stress components (e.g., language insufficiency, social isolation, discrimination) may combine to determine the physical activity of Chinese international students, and these should be addressed as a group of potential stressors.

Students feeling stressed because of their studies or transitions may recognize the mental health benefits of being physically active. For Chinese students in Canada, reducing stress related to academics as well as cross-cultural transitions was an important reason to be physically active (Brunette et al., 2011). Motivation to exercise to reduce stress was related to VPA, and SWB was related to more MPA in this study. It is likely that the relationship between SWB and MPA is reciprocal. Sport or exercise for stress reduction should focus on activities that induce physiological responses (e.g., social outings, dancing) because students can then identify the bodily sensations of stress and employ 
these strategies to minimize stress responses (Gómez, Urzúa, \& Glass, 2014). First year students may experience a large amount of stress compared to the older students, while senior students may be particularly concerned about their academic success, feelings of guilt towards their family, and experiences related to discrimination.

International students in the United States who lived in the host country longer experienced more discrimination (Poyrazli \& Lopez, 2007). This was explained by culture shock theory which describes that after a period of initial fascination and excitement, more negative aspects of living in the host culture were observed. There may also be an increase in understanding of verbal discrimination with improving language skills. Our finding that senior students experienced more discrimination may complement other research indicating that first year students in Canada experienced little discrimination on and off campus (Grayson, 2014). Research has shown that integration with the host culture could result in fewer experiences of discrimination (Berry \& Sabatier, 2010). Experiencing discrimination could discourage orientation to the host culture, however, making such a solution unappealing (Berry \& Sam, 2006). Students could learn effective coping strategies such as problem-focused coping including personal confrontation, taking formal actions, and support seeking which were found to reduce the distressing influence of discrimination for Koreans in Canada (Noh \& Kaspar, 2003). Emotion-focused coping (i.e., passive acceptance, emotional distraction) may also be effective, but only if a person feels supported in their cultural community (Noh \& Kaspar, 2003). If discrimination cannot be removed at the personal, community, or institutional level, international students should be equipped with coping strategies and connected with campus services and mentors to help in language learning and stress reduction (Frey \& Roysircar, 2006). Indeed, international students would benefit from such programs regardless of their experience of discrimination. Institutions could implement education campaigns about recognizing and avoiding discrimination (Poyrazli \& Lopez, 2007). Implementing these programs early in an international student's experience could help build resilience and allow students to focus on academic and personal growth.

\section{LIMITATIONS}

The design implemented in this study is limited due to potential differences that were not measured between the student groups. The self-efficacy measure may have been too specific to exercise participation rather than physical activity more generally. Self-reported physical activity may also be inaccurate due to participant recall errors or inaccuracy in reporting. The intention measure may not be fully representative of goal setting or self-regulation and is limited by examining intention to do future behavior cross-sectionally. Physical inactivity was not included as an outcome variable and could be examined in future research in understanding relationships between cognition and health behavior in Chinese international students.

\section{CONCLUSIONS}

This study showed that Chinese international students may perceive their environment differently, and experience different kinds of stress as they advance in their academic years. Walking behavior may also decline over time for these students. Developing self-efficacy and self-regulation strategies for health behaviors early in the post-secondary journey may be important to maintain these positive behaviors, especially when balancing them with academic and other commitments. Chinese international students who are active likely understand the stress relieving benefits of physical activity, but they might benefit from experiencing the social benefits as well. Practitioners should organize physical activity programming with year of study and cultural factors in mind. 


\section{REFERENCES}

Allen, J. T., Drane, D. D., Byon, K. K., \& Mohn, R. S. (2010). Sport as a vehicle for socialization and maintenance of cultural identity: International students attending American universities. Sport Management Review, 13(4), 421-434. https://doi.org/10.1016/j.smr.2010.01.004

Ayotte, B. J., Margrett, J. A., \& Hicks-Patrick, J. (2010). Physical activity in middle-aged and young-old adults: The roles of self-efficacy, barriers, outcome expectancies, self-regulatory behaviors and social support. Journal of Health Psychology, 15(2), 173-185. https://doi.org/10.1177/1359105309342283

Bai, J. (2016). Development and validation of the Acculturative Stress Scale for Chinese College Students in the United States (ASSCS). Psychological Assessment, 28(4), 443-447. https://doi.org/10.1037/pas0000198

Bandura, A. (1986). Social foundations of thought and action: A social cognitive theory. Englewood Cliffs, NJ, US: PrenticeHall, Inc.

Bandura, A. (1997). Self-efficacy: The exercise of control. New York: W.H. Freeman.

Bandura, A. (2004). Health promotion by social cognitive means. Health Education \& Behavior, 31(2), $143-164$. https://doi.org/10.1177/1090198104263660

Berry, J. W. (1997). Immigration, acculturation, and adaptation. Applied Psychology, 46(1), 5-34. https://doi.org/10.1111/j.1464-0597.1997.tb01087.x

Berry, J. W., \& Sabatier, C. (2010). Acculturation, discrimination, and adaptation among second generation immigrant youth in Montreal and Paris. International Journal of Intercultural Relations, 34(3), 191-207. https://doi.org/10.1016/j.ijintrel.2009.11.007

Berry, J. W., \& Sam, D. L. (2006). The cambridge handbook of acculturation psychology (J. W. Berry \& D. L. Sam, Eds.). Cambridge: Cambridge University Press.

Brunette, M. K., Lariviere, M., Schinke, R. J., Xing, X., \& Pickard, P. (2011). Fit to belong: Activity and acculturation of Chinese students. Journal of Sport Behaviour, 34(3), 201-227.

Chen, A., \& Liu, X. (2008). Expectancy beliefs and perceived values of Chinese college students in physical education and physical activity. Journal of Physical Activity and Health, 5, 262-274.

https://doi.org/10.1123/jpah.5.2.262

Cohen, S., Kamarck, T., \& Mermelstein, R. (1983). A global measure of perceived stress. Journal of Health and Social Behavior, 24(4), 385. https://doi.org/10.2307/2136404

Diener, E., Emmons, R. A., Larsen, R. J., \& Griffin, S. (1985). The Satisfaction With Life Scale. Journal of Personality Assessment, 49(1), 71-75. https://doi.org/10.1207/s15327752jpa4901_13

Diener, E., \& Ryan, K. (2009). Subjective well-being: A general overview. South African Journal of Psychology, 39(4), 391-406. https://doi.org/10.1177/008124630903900402

Doerksen, S. E., Umstattd, M. R., \& McAuley, E. (2009). Social cognitive determinants of moderate and vigorous physical activity in college freshmen. Journal of Applied Social Psychology, 39(5), 1201-1213. https://doi.org/10.1111/j.1559-1816.2009.00478.x 
Egli, T., Bland, H. W., Melton, B. F., \& Czech, D. R. (2011). Influence of age, sex, and race on College students' exercise motivation of physical activity. Journal of American College Health, 59(5), 399-406. https://doi.org/10.1080/07448481.2010.513074

Faul, F., Erdfelder, E., Lang, A.-G., \& Buchner, A. (2007). G*Power 3: A flexible statistical power analysis program for the social, behavioral, and biomedical sciences. Behavior Research Methods, 39(2), 175-191. https://doi.org/10.3758/BF03193146

Field, A. (2013). Discovering Statistics using IBM SPSS Statistics. California: Sage.

Frey, L. L., \& Roysircar, G. (2006). South Asian and East Asian international students' perceived prejudice, acculturation, and frequency of help resource utilization. Journal of Multicultural Counseling and Development, 34(4), 208-222. https:// doi.org/10.1002/j.2161-1912.2006.tb00040.x

Gerber, M., Barker, D., \& Pühse, U. (2012). Acculturation and physical activity among immigrants: a systematic review. Journal of Public Health, 20(3), 313-341. https://doi.org/10.1007/s10389-011-0443-1

Gómez, E., Urzúa, A., \& Glass, C. R. (2014). International student adjustment to college: social networks, acculturation, and leisure. Special Issue: Leisure and Transitions over the Lifespan, 32(1), 7-25.

Grayson, P. J. (2014). Negative racial encounters and academic outcomes of international and domestic students in four Canadian universities. Journal of International Students, 4(3), 262-278.

Guo, Q., \& Ross, C. M. (2014). An exploratory study of Asian international students' campus recreational sports participation. Recreational Sports Journal, 38(1), 55-68.

Ha, A. S., Macdonald, D., \& Pang, B. O. H. (2010). Physical activity in the lives of Hong Kong Chinese children. Sport, Education and Society, 15(3), 331-346. https://doi.org/10.1080/13573322.2010.493313

Hashim, H. A. (2012). Perceived barriers to recreation sport participation in university students: A comparison between international and local students in the United States. Pertanika Journal of Social Science and Humanities, 20(1), 197-203.

Hudson, S., Walker, G. J., Simpson, B., \& Hinch, T. (2013). The influence of ethnicity and self-construal on leisure constraints. Leisure Sciences, 35(2), 145-166. https://doi.org/10.1080/01490400.2013.761906

Huynh, Q.-L., Howell, R. T., \& Benet-Martinez, V. (2009). Reliability of bidimensional acculturation scores: A meta-analysis. Journal of Cross-Cultural Psychology, 40(2), 256-274. https://doi.org/10.1177/0022022108328919

Ito, E. (2014). The effects of culture on conceptualizations of leisure, control, and positive affect between Japanese and Canadian undergraduate students (University of Alberta). https://doi.org/10.7939/R3PN8XP8N

Karoly, P. (1993). Mechanisms of self-regulation: A systems view. Annual Review of Psychology, 44(1), $23-52$. https://doi.org/10.1146/annurev.ps.44.020193.000323

Li, S., \& Zizzi, S. (2018). A case study of international students' social adjustment, friendship development, and physical activity. Journal of International Students, 8(1), 389-409. https://doi.org/10.32674/jis.v8i1.171 
Luszczynska, A., \& Schwarzer, R. (2005). Social Cognitive Theory. In M. Connor \& P. Norman (Eds.), Predicting health behaviour (2nd ed., pp. 127-169). New York: Open University Press.

Macfarlane, D. J., Lee, C. C. Y., Ho, E. Y. K., Chan, K. L., \& Chan, D. T. S. (2007). Reliability and validity of the Chinese version of IPAQ (short, last 7 days). Journal of Science and Medicine in Sport, 10(1), 45-51. https://doi.org/10.1016/j.jsams.2006.05.003

Marín, G., \& Marín, B. (1991). Research with Hispanic Populations. https://doi.org/10.4135/9781412985734

Markland, D., \& Ingledew, D. K. (1997). The measurement of exercise motives: Factorial validity and invariance across gender of a revised Exercise Motivations Inventory. British Journal of Health Psychology, 2(4), 361-376. https://doi.org/10.1111/j.2044-8287.1997.tb00549.x

Markus, H. R., \& Kitayama, S. (1991). Culture and the self: Implications for cognition, emotion, and motivation. Psychological Review, 98(2), 224-253. https://doi.org/10.1037/0033-295X.98.2.224

McLachlan, D. A., \& Justice, J. (2009). A grounded theory of international student well-being. Journal of Theory Construction \& Testing, 13(1), 27-32.

Noh, S., \& Kaspar, V. (2003). Perceived discrimination and depression: moderating effects of coping, acculturation, and ethnic support. American Journal of Public Health, 93(2), 232-238.

https://doi.org/10.2105/AJPH.93.2.232

Park, J., \& Kitayama, S. (2012). Interdependent selves show face-induced facilitation of error processing: Cultural neuroscience of self-threat. Social Cognitive and Affective Neuroscience, nss125.

https://doi.org/10.1093/scan/nss125

Poyrazli, S., \& Lopez, M. D. (2007). An exploratory study of perceived discrimination and homesickness: A comparison of international students and American students. The Journal of Psychology, 141(3), 263-280. https://doi.org/10.3200/JRLP.141.3.263-280

Rodgers, W. M., Wilson, P. M., Hall, C. R., Fraser, S. N., \& Murray, T. C. (2008). Evidence for a multidimensional self-efficacy for exercise scale. Research Quarterly for Exercise and Sport, 79(2), 222-234. https://doi.org/10.1080/02701367.2008.10599485

Rovniak, L. S., Anderson, E. S., Winett, R. A., \& Stephens, R. S. (2002). Social cognitive determinants of physical activity in young adults: A prospective structural equation analysis. Annals of Behavioral Medicine, 24(2), 149_ 156. https://doi.org/10.1207/S15324796ABM2402_12

Ryder, A. G., Alden, L. E., \& Paulhus, D. L. (2000). Is acculturation unidimensional or bidimensional? A head-tohead comparison in the prediction of personality, self-identity, and adjustment. Journal of Personality and Social Psychology, 79(1), 49-65. https://doi.org/10.1037/0022-3514.79.1.49

Sachs, J. (2003). Validation of the satisfaction with life scale in a sample of Hong Kong university students. Psychologia, 46(4), 225-234. https://doi.org/10.2117/psysoc.2003.225

Sallis, J. F., Grossman, R. M., Pinski, R. B., Patterson, T. L., \& Nader, P. R. (1987). The development of scales to measure social support for diet and exercise behaviors. Preventive Medicine, 16(6), 825-836. https://doi.org/10.1016/0091-7435(87)90022-3 
Shim, G., Freund, H., Stopsack, M., Kämmerer, A., \& Barnow, S. (2014). Acculturation, self-construal, mental and physical health: An explorative study of East Asian students in Germany. International Journal of Psychology, 49(4), 295-303. https://doi.org/10.1002/ijop.12008

Stults-Kolehmainen, M. A., \& Sinha, R. (2014). The effects of stress on physical activity and exercise. Sports Medicine, Vol. 44. https://doi.org/10.1007/s40279-013-0090-5

Tsai, J. L., Knutson, B., \& Fung, H. H. (2006). Cultural variation in affect valuation. Journal of Personality and Social Psychology, 90, 288-307. https://doi.org/10.1037/0022-3514.90.2.288

Wang, Z., Chen, J., Boyd, J. E., Zhang, H., Jia, X., Qiu, J., \& Xiao, Z. (2011). Psychometric roperties of the Chinese version of the perceived stress scale in policewomen. PLOS ONE, 6(12), e28610. https://doi.org/10.1371/journal.pone.0028610

Ward, C., \& Kennedy, A. (1999). The measurement of sociocultural adaptation. International Journal of Intercultural Relations, 23(4), 659-677. https://doi.org/10.1016/S0147-1767(99)00014-0

Yan, Z., \& FitzPatrick, K. (2016). Acculturation and health behaviors among international students: A qualitative approach. Nursing \& Health Sciences, 18(1), 58-63. https://doi.org/10.1111/nhs.12232

Yeh, C. J., \& Inose, M. (2003). International students' reported English fluency, social support satisfaction, and social connectedness as predictors of acculturative stress. Counselling Psychology Quarterly, 16(1), 15-28. https://doi.org/10.1080/0951507031000114058

Yoh, T., Yang, H., \& Gordon, B. (2008). Status of participation in physical activity among international students attending colleges and universities in the United States. College Student Journal, 42(4), 1110-1117.

Zheng, X., \& Berry, J. W. (1991). Psychological adaptation of Chinese sojourners in Canada. International Journal of Psychology, 26(4), 451-470. https://doi.org/10.1080/00207599108247134

Author correspondance may be addressed to:

Kimberley D. Curtin, Ph.D.

Postdoctoral Fellow

Policy, Location and Access in Community Environments (PLACE) Research Lab

School of Public Health

University of Alberta

3-294 Edmonton Clinic Health Academy

11405 - 87 Ave

Edmonton, AB T6G 1C9

kcurtin@ualberta.ca 


\section{Author's Note and Acknowledgements}

We could like to acknowledge Dr. Jinjing Gui, Dr. Chen Chen, and Nanxi Yan for their help in translating the surveys and data collection.

The first author was supported by a Social Sciences and Humanities Research Council Joseph-Armand Bombardier Doctoral Scholarship. This source had no involvement in study design, data analysis, data collection, data interpretation or report writing. 